\title{
Meta-analytic structural equation modeling made easy: A tutorial and web application for one-stage MASEM
}

\author{
Suzanne Jak ${ }^{1}$ ( ) Hongli Li $^{2}$ | Laura Kolbe $^{1}$ | Hannelies de Jonge ${ }^{1}$ | \\ Mike W.-L. Cheung ${ }^{3}$ ()
}

${ }^{1}$ Research Institute of Child Development and Education, University of Amsterdam, Amsterdam, The Netherlands

${ }^{2}$ Department of Educational Policy Studies, Georgia State University, Atlanta, Georgia, USA

${ }^{3}$ Department of Psychology, National University of Singapore, Singapore

\section{Correspondence}

Suzanne Jak, Research Institute of Child Development and Education, University of Amsterdam, Nieuwe Achtergracht

127, 1018 WS Amsterdam, The

Netherlands.

Email: s.jak@uva.nl

\section{Funding information}

Dutch Research Council, Grant/Award Number: NWO-VENI-451-16-001

\begin{abstract}
Meta-analytic structural equation modeling (MASEM) refers to fitting structural equation models (SEMs) (such as path models or factor models) to metaanalytic data. Currently, fitting MASEMs may be challenging for researchers that are not accustomed to working with R software and packages. Therefore, we developed webMASEM; a web application for MASEM. This app implements the one-stage MASEM approach, and allows users to apply MASEM in a user-friendly way. The aim of this article is to provide a tutorial on one-stage MASEM and a practical guide to webMASEM. We will pay specific attention to how the data should be structured and prepared for webMASEM, because mistakes in this step may lead to faulty results without receiving an error message. The use of webMASEM is illustrated with an analysis of a meta-analytic path model in which the path coefficients are moderated by a study-level variable, a meta-analytic factor model in which the factor loadings are moderated by a study-level variable, and a meta-analytic panel model in which the effects are moderated by a study-level variable. All used datafiles and R scripts are available online.
\end{abstract}

\section{K E Y W O R D S}

MASEM, meta-analytic structural equation modeling, moderator analysis, one-stage MASEM, shiny app

\section{Highlights}

\section{What is already known}

- Meta-analytic structural equation modeling is a useful tool to fit structural equation models (SEMs) to meta-analytic datasets

- Researchers may have hypothesis about how the SEM parameters may be a function of study-level moderator variables

- One-stage MASEM is a relatively new method that allows for flexible moderator analyses 


\section{What is new}

- We provide a didactic discussion of the features of one-stage MASEM, as well as a user-friendly web application

- This article shows how the dataset should be prepared and structured for webMASEM (and for analysis using the metaSEM package directly)

- All R scripts and datasets are available online

Potential impact for Research Synthesis Methods readers outside the authors' field

- The tutorial and online application make applying MASEM easier for researchers in all fields

\section{1 | INTRODUCTION}

Meta-analytic structural equation modeling (MASEM) refers to fitting structural equation models (SEMs) to meta-analytic data using correlation matrices. This article serves as a primer on one-stage MASEM and as a tutorial for the associated Shiny app "webMASEM," which is built on functions from the metaSEM package ${ }^{1}$ and the semPlot package. ${ }^{2}$ The Shiny app is available through https://sjak.shinyapps.io/webMASEM/. We focus on onestage MASEM, because this method is currently one of the most flexible MASEM methods for moderation analysis. Other proposed methods are, for example, the GLSmethod, ${ }^{3-5}$ two-stage SEM, ${ }^{6-8}$ Bayesian MASEM, ${ }^{9}$ and univariate MASEM. ${ }^{10}$ For a detailed discussion of the possibilities and differences between these MASEM methods, see Jak and Cheung. ${ }^{11}$

Our goal is to provide enough information to conceptually understand what happens behind the scenes when using webMASEM, while leaving the more technical details to be read elsewhere. ${ }^{11}$ We will provide specific guidance in how the dataset should be prepared before the MASEM analysis because doing it incorrectly may lead to faulty results without receiving an error message. Moreover, we will provide detailed examples of the evaluation of two path models and a factor model.

\section{1 | What kind of research questions can be answered with one-stage MASEM?}

One-stage MASEM is an appropriate technique when one wants to test a SEM, such as a path model, on the averaged correlations between research variables across several independent studies. Most studies applying MASEM evaluate path models (e.g., van Dijk et $\mathrm{al}^{12}$ ) or factor analytic models (e.g., Agelink van Rentergem et $\mathrm{al}^{13}$ ), but full SEMs that combine factor analysis and path analysis can also be evaluated (e.g., Bresin ${ }^{14}$ ). Besides testing the overall theoretical model, one can also evaluate whether the SEM parameters (e.g., path coefficients) vary with study-level moderators, such as study quality, the type of sample investigated (e.g., patient or community sample), or the country where the study was conducted. For example, the factor loadings of the Hospital Anxiety and Depression Questionnaire (HADS) may be higher for patient samples than for community samples. ${ }^{15}$ Or in a path model, the unique effect of behavioral intentions on behavior may be stronger in adult samples than in adolescent samples. ${ }^{16}$

\section{2 | What is one-stage MASEM?}

One-stage MASEM is a random-effects technique, meaning that it is assumed that each study has its own specific population correlation matrix. The differences between the population correlation matrices are modeled by estimating a matrix with between-study variances and covariances. These variances are estimated using a model matrix that is often denoted " $\mathbf{T}^{2}$ " ("Tau squared"). $\mathbf{T}^{2}$ is commonly specified as diagonal, meaning that the covariances are assumed zero. The SEM model of interest is imposed on the average correlation matrix across studies. One of the criticisms of the random effects model is that the average correlation matrix may not be the real correlation matrix in any of the studies. ${ }^{17}$ Whether it is informative to evaluate the average correlations across studies depends on the type of samples and materials used in the primary studies included in the meta-analysis. Therefore, researchers planning on doing a MASEM analysis should set the inclusion criteria such that the information obtained by fitting a SEM on the average correlations across studies will be meaningful, or such that the heterogeneity across studies can be investigated. Becker and Aloe ${ }^{5}$ provide a discussion of the key points of the formulation of research questions, the search process, and inclusion criteria relevant for conducting a MASEM analysis. 
With one-stage MASEM, study-level moderator variables can be used to explain study-level heterogeneity in SEM parameters. The specific advantage of one-stage MASEM over other MASEM methods is that the moderating study-level variables can be dichotomous as well as continuous variables. For two-stage SEM, one needs to categorize continuous study-level moderators in order to apply subgroup analysis. ${ }^{18}$ However, categorization of variables leads to a loss of information and associated statistical power, as well as difficulty choosing an appropriate cutting point. Categorization should therefore be avoided whenever possible. ${ }^{19}$

\section{3 | Data needed for one-stage MASEM}

The minimum required data for MASEM are the bivariate correlation coefficients between the variables of interest, obtained from several independent samples, and the sample sizes. For moderation analysis one also needs to code the values on the study-level moderator variable for each sample.

One often asked question is how many studies/samples are needed for MASEM. Since the minimum number of studies needed depends on many factors (such as the number of variables, type of the model, complexity of the model, correctness of the model, size of the betweenstudy variances, and percentage of missing data), it is difficult to provide exact advice. The simulations based on the analysis of a factor model using one-stage MASEM have shown that analyzing 10 samples led to adequate parameter estimates, but incorrect standard errors for a two-factor model with five indicators. ${ }^{11}$ Analyzing 30 samples was enough to obtain adequate parameter estimates and standard errors. The simulation study did not contain conditions with number of samples between 10 and 30. Note that these results are based on a limited simulation study, so they will not apply to all MASEM models or all research settings. Future simulation studies may be conducted to evaluate the minimum number of studies needed for adequate performance of one-stage MASEM with other types of models and in other situations.

The number of bivariate correlation coefficients that can be coded from the sample may differ across studies. In general, the number of unique correlations in a correlation matrix of $p$ variables equals $p \times(p-1) / 2$. Suppose that the model of interest contains six variables, being three predictor variables, two mediating variables, and one outcome variable. The number of bivariate correlations between the variables of interest then equals $(6 \times 5) / 2=15$. In the ideal situation, a researcher would code 15 bivariate correlation coefficients from each study included in the synthesis. In reality, many studies in the synthesis will include only part of the variables of interest. For example, a specific study may only have included one predictor, one mediator, and the outcome variable. In this case, the study contributes three correlations instead of 15 . Another study may have only included the two mediator variables, therefore contributing only one correlation. Yet another study may have included all variables of interest except for one predictor, leading to 10 bivariate correlations to be reported.

Besides studies missing correlations on the variable level, some studies may just not report all correlations between the included variables, leading to missingness on the correlation level. For example, a primary study may report the correlations between predictor variables and an outcome variable, but not between the predictor variables itself. Both types of missingness, on the variable level and on the correlation level, are technically not a problem for a MASEM to be applied. However, if the missingness is not missing at random (MAR), or missing completely at random (MCAR), for example, because specifically the relatively low correlation coefficients are not reported, then the results from the MASEM will be biased. ${ }^{11,20}$ In fact, this is not different for MASEM than for other meta-analytic techniques, where publication bias is a serious concern. The solution for researchers so far is therefore to include as many appropriate but unpublished studies as possible in the MASEM analysis. It is the hope that preregistration of studies will lead to better meta-analytic results in the future. ${ }^{21}$

In order to fit a SEM model, one needs data on all bivariate correlations between the variables in the SEM. Quite often, some of the correlations are not of direct interest to the researcher(s) doing the synthesis. For example, researchers sometimes did not code the correlations between predictor variables in a path model, to only discover that those correlations were needed when trying to run the MASEM analysis. Therefore, it is important to have a clear understanding of the variables of interest and the hypothetical model(s), and to code all correlations between the variables of interest.

\subsection{Organization of the dataset}

When coding the correlation coefficients, the most convenient form is to have a coding spreadsheet with one row for each independent sample and one column for each pair of variables. This means that with $p$ variables of interest, one has to create $p \times(p-1) / 2$ columns to code the bivariate correlations. Furthermore, one needs a column with sample sizes per study and possibly several other columns with other study-level information. 
Missing values for correlation coefficients should be coded NA (which means "not available" in R) when one uses the metaSEM package directly for the analysis. If one uses webMASEM, either a blank space or NA will be recognized as a missing value.

For the bivariate correlations, it is important to pay attention to the ordering of the columns, because they have to be in a specific order for the analysis. That is, the rows with the correlation coefficients in each study will be transformed to a list of the study's correlation matrices. One has to make sure that the correlation coefficients are placed in the correct position in the matrices. The $\mathrm{R}$ functions used by webMASEM fill the matrices columnwise, which is known as column-major order.* This process is illustrated in Figure 1, showing a hypothetical model with four variables of interest. The researcher decides to use the following order of the variables: X1, $\mathrm{X} 2, \mathrm{M}$, and $\mathrm{Y}$. This means that a correlation matrix of these four variables will look like the one in the center of Figure 1. The six correlation coefficients in this matrix are labeled column-wise, using $\mathrm{c} 1$ to $\mathrm{c} 6$. The dataset with the correlation coefficients for each study in one row should be in this exact order. The first correlation coefficient should be the correlation between X1 and X2 (c1), the second correlation coefficient should be the correlation between $\mathrm{X} 1$ and $\mathrm{M}$ (c2), the third should be the correlation between $\mathrm{X} 1$ and $\mathrm{Y}(\mathrm{c} 3)$, the fourth should be the correlation between $\mathrm{X} 2$ and $\mathrm{M}$ (c4) and so on. Users do not need to provide the variances, which will always be one since one-stage MASEM analyzes correlation matrices. Although this procedure seems quite obvious, we experience that researchers applying MASEM, quite often

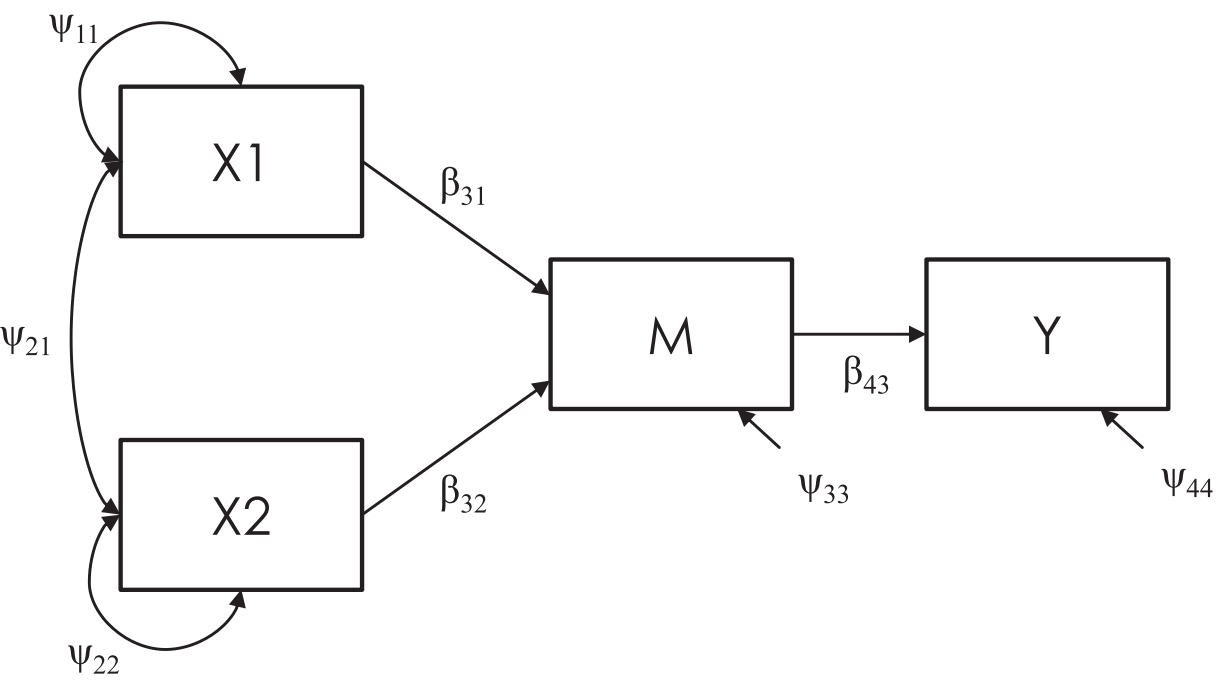

Order of the variables: X1, X2, M, Y

FIGURE 1 An illustration of how the order of varibles determines the ordering of the columns in the coding sheet
Structure of the correlation matrix

\begin{tabular}{|l|c|c|c|c|}
\hline & $\mathbf{X} 1$ & $\mathbf{X}$ & $\mathbf{M}$ & $\mathbf{Y}$ \\
\hline $\mathbf{X} 1$ & 1 & & & \\
\hline $\mathbf{X} 2$ & $\mathrm{c} 1$ & 1 & & \\
\hline $\mathbf{M}$ & $\mathrm{c} 2$ & $\mathrm{c} 4$ & 1 & \\
\hline $\mathbf{Y}$ & $\mathrm{c} 3$ & $\mathrm{c} 5$ & $\mathrm{c} 6$ & 1 \\
\hline
\end{tabular}

\begin{tabular}{|c|c|c|c|c|c|c|c|}
\hline \multirow{6}{*}{$\begin{array}{l}\text { Structure of } \\
\text { the dataset }\end{array}$} & \multicolumn{2}{|r|}{ Cl } & C2 & c3 & c4 & c5 & c6 \\
\hline & Study & $\times 2 \times 1$ & $M \_1$ & $Y \_1$ & $M \_2$ & $Y \_X 2$ & $Y_{-} \mathbf{M}$ \\
\hline & 1 & & & & .22 & .34 & .29 \\
\hline & 2 & .10 & & .14 & & .14 & \\
\hline & 3 & & & & .23 & .13 & .09 \\
\hline & 4 & & & & & & \\
\hline & & & & & & & \\
\hline
\end{tabular}


place correlation coefficients in different positions of the matrix than intended. The danger of making such a mistake is that it leads to completely incorrect results, possibly without any error or warning.

\section{5 | Specifying the SEM model in lavaan syntax}

The easiest way to specify the SEM is to use the syntax of the $\mathrm{R}$ package lavaan. ${ }^{22}$ The lavaan syntax will then be converted to the necessary matrices using a function in the metaSEM package. ${ }^{1}$ The lavaan syntax is relatively straightforward and is explained in a tutorial on lavaan's website: http://lavaan.ugent.be/tutorial/syntax1.html. The model is specified by referring to the variable names and using different operators to define how the variables are related. In Figure 1 for example, variables $\mathrm{X} 1$ and $\mathrm{X} 2$ are hypothesized to affect $\mathrm{Y}$ only indirectly through $\mathrm{M}$, representing full mediation of the effects of $\mathrm{X} 1$ and $\mathrm{X} 2$. The lavaan syntax for this model is:

$\mathrm{M} \sim \mathrm{X} 1+\mathrm{X} 2$
$\mathrm{Y} \sim \mathrm{M}$
$\mathrm{X} 1 \sim \mathrm{X} 2$
$\mathrm{X} 1 \sim 1$ 1 X1
$\mathrm{X} 2 \sim \sim 1 * \mathrm{X} 2$
$\mathrm{M} \sim \mathrm{M}$
$\mathrm{Y} \sim \sim \mathrm{Y}$

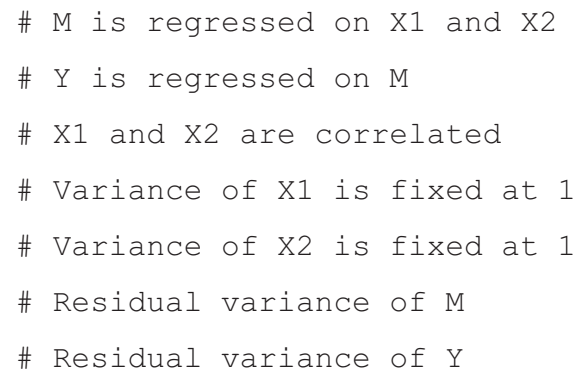

Here, the single operator means "is regressed on." So, $\mathrm{M}$ is regressed on $\mathrm{X} 1$ and $\mathrm{X} 2$. This means that $\mathrm{X} 1$ and $\mathrm{X} 2$ have a direct effect on $\mathrm{M}$, or, the arrows go from $\mathrm{X} 1$ and $\mathrm{X} 2$ to $\mathrm{M}$. One common mistake is to read the $\sim$ as if it means "has a direct effect on," which leads to a path model with direct effects that are exactly the other way around than intended.

The operator represents covariance. A covariance of a variable with itself is a variance. So, $\mathrm{X} 1 \sim \sim \mathrm{X} 2 \mathrm{spec}-$ ifies that the two predictor variables are correlated, while $\mathrm{M} \sim \sim \mathrm{M}$ specifies the residual variance of $\mathrm{M}$, as $\mathrm{M}$ is not an independent variable. In one-stage MASEM, we are analyzing correlation matrices instead of covariance matrices. This has a consequence for the estimates of variances in the model, because the model implied variance for each variable is one by definition. The metaSEM package has built-in functionality to take this issue into account, but requires the variances of independent variables to be fixed at 1 . In the example syntax, X1 1*X1 fixes the variance of $\mathrm{X} 1$ at 1 .
If the multiplication sign * is preceded by a number, then the parameter is fixed at that number. If the ${ }^{*}$ sign is preceded by a character, then the parameter is still freely estimated, but the parameter gets a label. For example, $\mathrm{M} \sim \mathrm{b} 31 * \mathrm{X} 1+\mathrm{b} 32 * \mathrm{X} 2$, would label the respective regression coefficient "b31" and "b32." Labeling parameters can be convenient because the labels will also be used in the output of the analysis. Also, labeling parameters is necessary if one wants to test functions of parameters, such as indirect effects. A third function of parameter labels is that if you provide the same label to two or more parameters, these parameters will be constrained to be equal.

Latent variables, also called common factors in SEM, are specified in lavaan using the $=\sim$ operator. The name of the common factor should be on the left side of the operator, while the indicators of the factor should be on the right side. For example, the example code below provides the lavaan syntax for a two-factor model where each factor has three indicators:

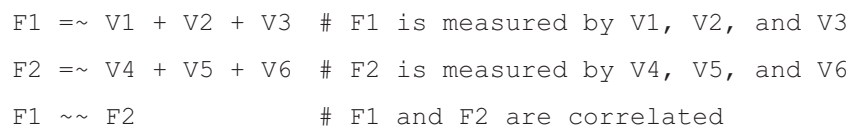

The first two lines specify that the first factor is measured by the respective indicators. The last line specifies that the common factors covary. The residual variances of the indicators will be included in the model automatically, even if one does not provide the parameters in the syntax.

Latent variables always need to be provided a scale by fixing one of the related parameters to a certain value. Common methods are fixing one factor loading per factor to one, or fixing the factor variance to one. In the metaSEM package, common factors will automatically be scaled by fixing the (possibly residual) factor variance to one, so there is no need to fix one of the factor loadings to one. In fact, the factor variance cannot be freed in metaSEM, so even when one would add F1 F1 in the syntax, the parameter will be fixed in the analysis.

One can write comments in the lavaan syntax using the hashtag symbol (\#). Any text that comes after the hashtag will be ignored when evaluating the syntax. Using comments in the syntax generally improves the readability of the code.

\subsection{How is the model fit to the data?}

In this section, we will briefly outline the more technical details of one-stage MASEM. In standard (not meta- 
analytic) SEM, the variances and covariances between the observed variables in a single dataset are modeled to be a function of SEM parameters. In one-stage MASEM, the average population correlations across studies are modeled to be a function of SEM parameters. The average correlations are based on a random-effects multivariate meta-analysis on correlation coefficients. ${ }^{8}$ So, conceptually, one-stage MASEM is an amalgamation of random-effects multivariate meta-analysis of correlation coefficients and SEM.

In its simplest form, the random-effects multivariate meta-analytic model decomposes the vector $\mathbf{r}_{k}$ of correlation coefficients for a study $k$ in three parts:

$$
\mathbf{r}_{k}=\boldsymbol{\rho}_{R}+\mathbf{u}_{k}+\boldsymbol{\varepsilon}_{k},
$$

where $\boldsymbol{\rho}_{R}$ indicates the vector of means of the population correlation coefficients over all studies, $\mathbf{u}_{k}$ is a vector of deviations of study $k$ 's population correlation coefficients from $\boldsymbol{\rho}_{R}$, and $\boldsymbol{\varepsilon}_{k}$ is a vector with the sampling deviations of study $k$. The covariances of $\mathbf{u}_{k}$ denote the betweenstudies covariance matrix, called $\mathbf{T}^{2}$ ("Tau squared"). The covariances of $\varepsilon_{k}$ represents the within-studies covariance matrix for study $k$, often denoted $\mathbf{V}_{k}$, which is first estimated for each study, and then treated as known in the final analysis. ${ }^{23}$ The SEM, for example a factor model, is nested under the model in Equation (1) and is obtained by restricting $\boldsymbol{\rho}_{R}$ :

$$
\boldsymbol{\rho}_{\mathrm{R}}=\operatorname{vechs}\left(\boldsymbol{\Lambda} \boldsymbol{\Phi} \boldsymbol{\Lambda}^{\mathrm{T}}+\boldsymbol{\Theta}\right),
$$

where $\boldsymbol{\Lambda}$ is a matrix with factor loadings, $\boldsymbol{\Phi}$ is a matrix with factor variances and covariances, and $\boldsymbol{\Theta}$ is a matrix with residual variances and covariances. The vechs() operator vectorizes the unique elements of its argument's lower-triangle elements in column-major order. That means although the model expression $\boldsymbol{\Lambda} \boldsymbol{\Phi} \boldsymbol{\Lambda}^{\mathrm{T}}+\boldsymbol{\Theta}$ leads to a model implied correlation matrix with number of rows equal to the number of observed variables in the model, applying the vechs() operator results in a vector with the same dimension as $\boldsymbol{\rho}_{\mathrm{R}}$.

\subsection{1 | Heterogeneity in one-stage MASEM}

It is important to notice that one-stage MASEM does not lead to an estimate of the between-studies variances of the SEM parameters itself. Instead, one-stage MASEM provides an estimate of the heterogeneity of the correlation coefficients. These correlation coefficients are an often complex function of the SEM model parameters. This is easier to grasp by looking at an example. We use
TA B LE 1 Model-implied correlation matrix of the path model in Figure 1

\begin{tabular}{lllll} 
& $\mathbf{X} 1$ & $\mathbf{X} 2$ & $\mathbf{M}$ & $\mathbf{Y}$ \\
$\mathrm{X} 1$ & 1 & & & \\
$\mathrm{X} 2$ & $\psi_{21}$ & 1 & & \\
$\mathrm{M}$ & $\beta_{31}+\beta_{32} \psi_{21}$ & $\beta_{31} \psi_{21}+\beta_{32}$ & 1 & \\
$\mathrm{Y}$ & $\beta_{31} \beta_{43}+\beta_{32} \beta_{43} \psi_{21}$ & $\beta_{31} \beta_{43} \psi_{21}+\beta_{32} \beta_{43}$ & $\beta_{43}$ & 1 \\
\hline
\end{tabular}

the path model on four variables depicted at the top part of Figure 1. This path model contains regression coefficients (indicated by $\beta$ 's) and a correlation between X1 and X2 (indicated by $\psi_{21}$ ). These parameters together form a model-implied correlation matrix between the four variables. The four by four model-implied correlation matrix for this example is provided in Table 1. Since we analyze correlation matrices, the diagonal elements of this matrix are always one and do not vary across studies. The off-diagonal elements represent the model-implied correlation coefficients. For example, the correlation between $\mathrm{X} 1$ and $\mathrm{Y}$ implied by this path model equals $\beta_{31} \beta_{43}+\beta_{32} \beta_{43} \psi_{21}$.

In one-stage MASEM, the between-studies covariance matrix, denoted $\mathbf{T}^{2}$, represents the between-studies covariances of these model-implied correlation coefficients. In this example, there are six model-implied correlation coefficients, so $\mathbf{T}^{2}$ will be a matrix with six rows and six columns. The diagonal elements of $\mathbf{T}^{2}$ contain between-studies variances and the off-diagonal elements represent between-studies covariances. In practice, it is often challenging to estimate the covariances in $\mathbf{T}^{2}$, because the number of elements is usually very large, possibly larger than the number of studies in the metaanalysis. For example, in our example there will be 15 off-diagonal elements in $\mathbf{T}^{2}$, but in models with five variables there will already be 45 between-studies covariances. Therefore, the $\mathbf{T}^{2}$ is usually restricted to be diagonal, so that between-studies variances of the correlation coefficients are estimated, but the between-studies covariances are fixed at zero. ${ }^{5}$ A diagonal $\mathbf{T}^{2}$ implies that the correlation coefficients are assumed to be independent at the population level, that is, the true effect sizes are independent. ${ }^{8}$ The sampling covariance between correlation coefficients from the same study is still taken into account by the within-studies covariance matrices.

\subsection{2 | Explaining heterogeneity with moderators}

The model can be extended by including study-level variables as predictors of the SEM parameters. For example, 
the direct effect of $\mathrm{M}$ on $\mathrm{Y}$ in Figure 1 could be modeled as a function of a study-level moderator variable $\mathrm{Z}$. The parameter $\beta_{43}$ then gets a subscript $k$ to indicate that it varies as a function of the study-level moderator variable $Z: \beta_{43 \mathrm{k}}=\beta_{43 \_0}+\beta_{43_{-} 1} \times Z_{\mathrm{k}}$. In this equation, $\beta_{43 \_0}$ represents the intercept, interpreted as the expected $\beta_{43}$ value for studies that score zero on the moderator variable. The parameter $\beta_{43 \_1}$ represents how $\beta_{43}$ is expected to change when the moderator variable increases by one unit. The parameters that will be estimated in one-stage MASEM are $\beta_{43 \_}$and $\beta_{43 \_1}$.

With one-stage MASEM, it cannot be evaluated how much of the variance in the SEM parameter itself is explained by a moderator variable. Instead, the moderating effect on the SEM parameter may explain part of the variance in the model-implied correlations. As shown in Table 1 , in our example path model, the parameter $\beta_{43}$ is part of the equations of the model-implied correlation between variables $\mathrm{X} 1$ and $\mathrm{Y}$, but also between variables $\mathrm{X} 2$ and $\mathrm{Y}$ and between variables $\mathrm{M}$ and $\mathrm{Y}$. Therefore, the moderation effect on this parameter may explain some of the between-studies variance in all three correlation coefficients. In principle, it can be quantified what proportion of the variance in each correlation coefficients is explained by the moderator variable (see Jak and Cheung ${ }^{11}$ ). However, given the complicated relationship between the SEM parameters and the model implied correlations, it is not clear how such proportions should be interpreted substantively.

Treating the SEM parameters as fixed, by not estimating their variances directly, is consistent with models in the standard SEM framework (e.g., Bauer ${ }^{24}$ ). An alternative approach is to treat the SEM parameters as random and estimate the model using a Bayesian method. ${ }^{9}$ Future studies may explore the advantages and disadvantages in addressing heterogeneity in MASEM with different methods.

All model parameters can be estimated with the osmasem() function in metaSEM, ${ }^{1}$ which implements full-information maximum likelihood estimation using the software OpenMx. ${ }^{25}$ Readers who want to read more on the details of the method can do so in Jak and Cheung. ${ }^{11}$

\section{2 | THE SHINY APP: webMASEM}

The application webMASEM is created using the package Shiny (Version 1.4.0.2) ${ }^{26}$ which facilitates the creation of web applications that interact with $\mathrm{R}$ (Version 4.0.1). ${ }^{27}$ For the analysis, webMASEM uses specific functions from the metaSEM package (Version 1.2.5). ${ }^{1}$ The plots of the models are created with semPlot (Version 1.1.2). ${ }^{2}$ The symbolic model implied covariance matrix is created with the symSEM package (Version 0.1). ${ }^{28}$ Users do not need to install $\mathrm{R}$ or any of these packages on their devices, as they can open webMASEM by clicking the link https:// sjak.shinyapps.io/webMASEM/. Alternatively, users can run webMASEM locally in their R session by running the full syntax provided on the Open Science Framework (OSF) for this article: https://osf.io/wh6d3/.

The application consists of four main tabs and a "Home" tab. The first tab provides an overview of some important concepts related to one-stage MASEM that should be understood before using the app, as well as references to this tutorial and the article about one-stage MASEM. ${ }^{11}$ The second tab lets users upload the dataset. The datafile should be a .txt, .dat, or .csv file with one row of information per study. The values should be separated by commas, tabs, or semicolons. If users just want to try webMASEM without supplying their own data, they can check the box that says "Choose dataset from metaSEM" and then select a dataset that is included in the metaSEM package. For ease of use, all fields that need user input have pre-filled values that match analysis of the dataset named "Roorda11.",29

The output section contains a tab "User dataset" showing the dataset as it was read in, a tab "Summary," showing a summary of the data (total number of studies, number of studies and total sample size per correlation coefficient) as well as the first six correlation matrices. The third output tab called "Average correlation matrix," can be used to estimate an average correlation matrix without fitting the SEM model. This average correlation matrix is estimated using a random-effects multivariate meta-analysis of the correlation matrix, as implemented with the tssem1()-function of the metaSEM package.

The third main tab "Overall analysis" facilitates fitting the SEM to the correlation matrices using onestage MASEM. The left panel of this tab allows the user to enter the lavaan syntax for the SEM. The page also contains a button that users can click to view the modelimplied correlation matrix based on the entered lavaan syntax.

If a user wants to evaluate an indirect effect, this is possible by ticking the checkbox that says "Test indirect effect" while naming the two direct effects that make up the indirect effect "beta1" and "beta2" in the lavaan syntax. The resulting indirect effect will equal beta1*beta2. Testing indirect effects will be illustrated in Example 1. The left panel also shows a "Rerun" button, which one can hit when the initial model estimation did not converge.

Below this, users can change the default settings of the functions from the metaSEM package that lie behind the Shiny app. Specifically, one can select the following options: 
1. The between-studies covariance matrix can be diagonal (the default), symmetrical, or zero. Selecting "zero" will result in fitting a common-effect (also called fixed-effects) model, which is hardly ever a realistic model. Selecting "symmetrical" means that all between-studies covariances will be estimated, which usually leads to estimation problems because there are too many parameters. Therefore, the default setting is recommended.

2. The confidence intervals shown in the summary can be likelihood-based confidence intervals ${ }^{30}$ or based on the standard errors (the default). Likelihood-based confidence intervals are recommended for the evaluation of indirect effects. ${ }^{31}$ The downside of likelihoodbased confidence intervals is that model estimation may take a long time.

3. The between-studies variances are transformed during estimation because very small or negative betweenstudies variances often cause computational problems. The default transformation (expLog) method is a log and exponential transformation. If one chooses "sqSD," the variances are estimated by squaring the standard deviations.

4. The starting values are the values for the between-studies variances from which the model estimation will start. The default is 0.05 , but one may select smaller or larger values. Choosing other starting values should not affect the final estimates, but adjusting starting values may improve convergence and save time.

The output panel will show a spinning bar while the estimation procedure is running (this may take several minutes for relatively large models). When the results are obtained, the user will see information about convergence, model fit, the summary as provided by the metaSEM package, as well as the back-transformed between-studies variances. The summary will show a column with the names of the parameters, three columns with information about the position of the parameter in the respective model matrix, followed by columns with the parameter estimates, associated standard errors, $\mathrm{z}$ values, and $p$ values. ${ }^{\dagger}$ The reported fit statistics are based on a $\chi^{2}$ value obtained by executing a likelihood ratio test between the hypothesized model for the correlation matrix and a saturated (unrestricted) model for the correlation matrix. Therefore, the fit indices refer to the fixed effects in the model, similar to the fit statistics obtained by analyzing the data with Two-Stage SEM. ${ }^{6}$

The fourth tab "Moderator analysis" facilitates testing moderating effects on one or more of the direct effects of the SEM by a study-level variable. The implementation in webMASEM is restricted to the evaluation of one moderator at a time (i.e., only one column of the dataset can be selected as the moderator variable). In the left panel of this tab, the user can select the moderator variable and the parameters that are to be tested for moderation. The advice is to limit the number of parameters selected to be moderated to the ones for which moderating effects are expected and enough studies contributed to the estimation of the parameter. ${ }^{11}$ One-stage MASEM will not be able to handle missing values on the moderator variable. Therefore, users will have to delete studies with missing values on the moderator from the dataset. The panel contains a checkbox to select whether the moderator should be centered and standardized before running the moderated one-stage MASEM. Centering and standardization of the moderator implies subtraction of the mean and division by the standard deviation for each value. Such a transformation generally improves the estimation of the model parameters and may facilitate the interpretation of the results for continuous moderator variables. For dummy variables (with only 0 and 1 values), centering and standardization will not be appropriate, nor needed.

The output for the moderation analysis will contain information about convergence, summary statistics of the moderator, an omnibus test for moderation (which is a multivariate test), the tests of the individual parameters (which are univariate tests), and the parameter estimates with associated statistics. For the moderation analysis, there will be no fit indices reported. The reason is that a saturated model does not exist for the model with moderating effects. Therefore we cannot evaluate the fit of the model with the moderator."

The last tab "Report" contains two buttons to generate MS Word files of the analyses that can be saved on the user's computer. The report will contain all information that is needed to reproduce the analyses, such as the values of the function arguments, as well as all the output that was created by webMASEM. We recommend users to make the datafile and this report available with publication of their study, so that the results are reproducible for readers.

In the next sections we present three example analyses using webMASEM, for which screenshots of the different pages of webMASEM are available as Data S1 for this manuscript. All datafiles for the illustration are available through the OSF page of this article (https://osf.io/wh6d3/), so readers can reproduce the results from this tutorial.

\section{1 | Example analysis 1}

We illustrate fitting a path model with the one-stage MASEM approach with webMASEM using a dataset from Roorda et al. ${ }^{33}$ This dataset was used to investigate whether (1) students' engagement acts as a mediator in the 


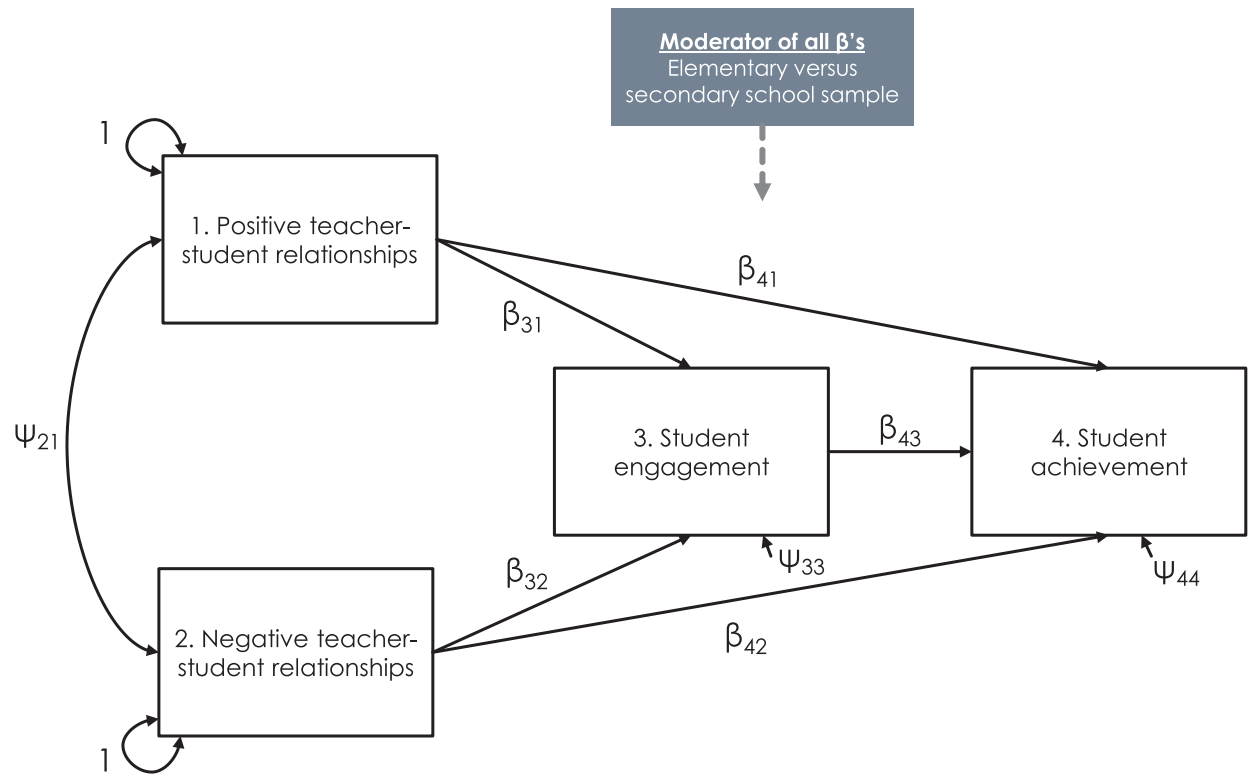

FIG URE 2 The research model from Roorda et al. ${ }^{29}$ [Colour figure can be viewed at wileyonlinelibrary.com]

association between affective teacher-student relationships and students' achievement and (2) whether the direct effects in the path model differed for primary and secondary school samples. The theoretical model of interest is shown in Figure 2.

Roorda et $\mathrm{al}^{33}$ gathered correlation coefficients between the four research variables, referred to as "pos" for positive teacher-student relationships, "neg" for negative teacher-student relationships, "enga" for engagament, and "achiev" for achievement, from 189 independent samples. Similar to the original data analysis, we exclude 10 of these samples because they contained students from both primary and secondary school or were not clear about whether their students were from primary schools or middle/junior high schools. Throughout all example analyses, we will use an unadjusted significance level of $\alpha=0.05$ for significance tests on SEM parameters and for the evaluation of moderating effects.

\subsection{1 | Example 1 - Data input}

The data were stored in a tab-delimited file, named "Data_Roorda.dat," which was selected after clicking the "Browse" button in the left panel (see screenshot 1.1 in Data S1). We selected that the file contains column names by ticking the box "Header" and selected "Tab" as the separator sign. Next, we need to enter the number of variables in the model (four in this example) and enter the variable names in the order that corresponds to the ordering of the correlation coefficients in the datafile as explained before. The variable names should be separated with commas.

The application will read in the columns from the selected datafile and repeat the column names under
"Select columns with the correlations." If the datafile does not include column names (so "Header" should be unchecked) the columns will be named v1, v2, v3, etc. In this example we select the six columns that contain the correlations between the four variables. Below this part (not shown in the print screen) we should also select the column with the sample sizes and the column with the (optional) moderating variable, in this example "primary_vs_secondary." If one then clicks the button "Update view user dataset" at the bottom of the left panel, the dataset as it was read in will appear in the right panel. Users can use this view to verify that the data were read in correctly, that is, that the correct columns were selected.

The output panel contains a subtab called "Summary." Selecting this tab will show some summary information from the uploaded dataset (see screenshot 1.2 in Data S1). First, it shows the variable names as entered at the left side and the variables in the dataset that will be used for onestage MASEM. The dataset for one-stage MASEM will always include a list with the correlation matrices, called "data," and a vector with sample sizes, called "n." If one also selects a moderator variable, that variable is also added to the dataset. In our output, we see that the dataset includes a list of 179 correlation matrices, a vector of 179 sample sizes, and a vector of 179 values on the variable "primary_versus_secondary."

Next, the summary shows the number of studies that included each variable on the diagonal and the number of studies that included each correlation coefficient off-diagonal. For example, 171 studies included the variable "pos" and 57 studies included the variable "neg." The correlation between "pos" and "neg" was reported in 43 studies and the correlation between "pos" and "achiev" was reported in 113 
studies. Below this table, the total sample size per variable and correlation are displayed in the same format. For example, the sum of the sample sizes of each study that included the correlation between "pos" and "neg" is 30,824. Below this, the first six correlation matrices are printed to give the user a sense of what the list of correlation matrices looks like. As a preliminary analysis, one could optionally estimate the average correlation matrix using a multivariate meta-analysis of the correlation matrices in the third tab of the output. When one pushes the button "Estimate average correlations," webMASEM will fit Stage 1 of the Two Stage SEM, using a diagonal between-studies variance matrix. This may take several minutes for larger correlation matrices. After verifying that the data were read in correctly, one can go to the next tab to fit the SEM to the data.

\subsection{2 | Example 1 - Overall analysis}

The lavaan syntax for the hypothesized path model is provided below. In the "Overall analysis" tab, we enter this lavaan syntax and then hit the button "Run Analysis" (see screenshot 1.3 in Data S1).

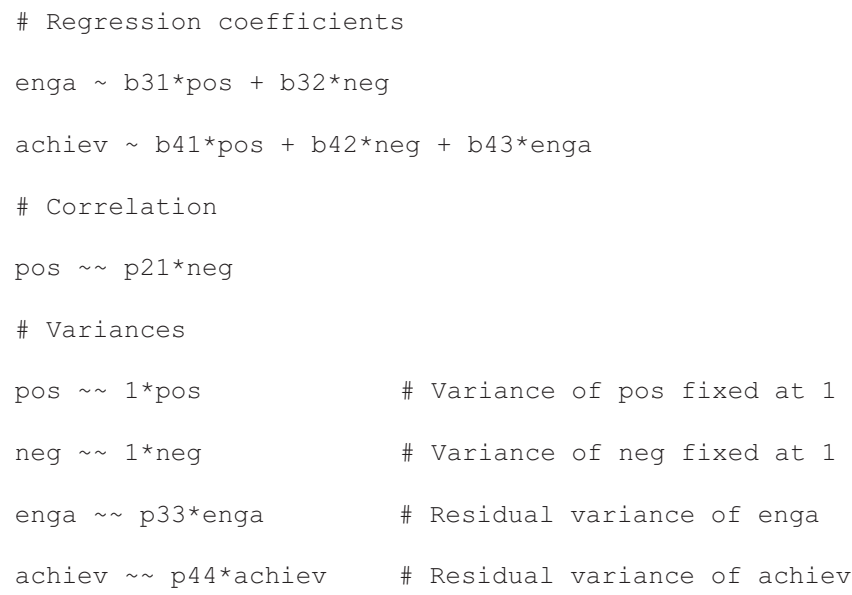

When the model estimation is done (this may take several minutes for larger models), we see a plot of the model with parameter estimates in the output panel. Below that, we see the model fit statistics for overidentified models (with degrees of freedom $>0$ ). Since this model has zero degrees of freedom, the fit statistics are not informative, but the parameter estimates, which are provided below this, are. Because the input for onestage MASEM are correlation matrices, which implies standardized observed variables, all SEM parameters obtained from the overall analysis are in standardized metric. If the user provides labels to the parameters in the lavaan model, then these labels will be used in the output. If the user does not provide parameter labels, the names of the parameters are constructed by connecting the variable names with "ON" for regression coefficients and "WITH" for (co)variances. For example, the direct effect of "enga" on "achiev" is called "achievONenga," because "achiev" is regressed ON "enga." In order to see how the SEM parameters together form the model-implied correlation matrix one could click the "View model implied matrix" button. Since the correlations can be a very complicated and long function of SEM parameters, this matrix is most readable if the parameter labels are short.

In the output, we see a positive direct effect of "pos" on "enga" ( $\left.\hat{\beta}_{31}=0.275, p<0.001\right)$, a negative effect of "neg" on "enga" ( $\left.\hat{\beta}_{32}=-0.193, p<0.001\right)$, and a positive effect of "enga" on "achiev" $\left(\hat{\beta}_{43}=0.233, p<0.001\right)$. The direct effects in meta-analytic path models can be interpreted as standardized regression coefficients. The indirect effects of "pos" and "neg" on "achiev" equal the product of the two direct effects that make up the indirect effects. One can obtain a 95\% likelihood-based confidence interval for an indirect effect by checking the box in the left panel below the lavaan input named "Test indirect effect" and naming the two direct effects that make up the indirect effect "beta1" and "beta2" (see screenshot 1.4 in Data S1). In order to test the indirect effect of "pos" on "achiev" in this example, the part of the lavaan syntax that specifies the direct effects becomes as follows.

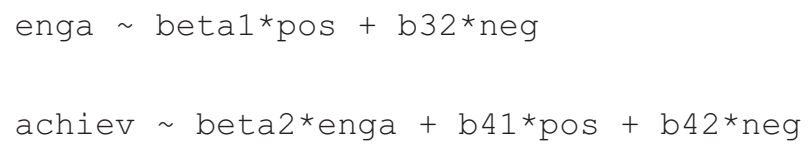

By checking the "Test indirect effect" box, one will request likelihood-based confidence intervals for all (constrained and free) parameters and the indirect effect. The indirect effect will be the last one in the long list provided in the output. In this example, the indirect effect of "pos" on "achiev" is 0.064 , with $95 \%$ likelihood-based confidence interval running from 0.047 to 0.082 . In order to evaluate the indirect effect of "neg" on "achiev," we need to switch the "beta1" label to the direct effect of "neg" on "enga," so that the lavaan code specifying the regression coefficients will be as follows and run the model again.

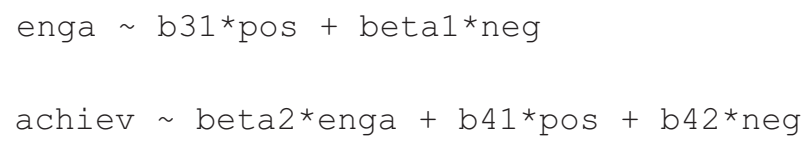

The output will then show that the indirect effect of "neg" on "achiev" equals -0.045 , with $95 \%$ likelihood- 
based CI $[-0.061 ;-0.031]$. Both indirect effects are statistically significant and of medium size ${ }^{\S}$ relative to effect sizes typically found in psychological research. ${ }^{34}$ The direct effects of "pos" and "neg" on "achiev" are also statistically significant $\left(\hat{\beta}_{41}=0.074, p<0.001\right.$ and $\hat{\beta}_{42}=$ $-0.063, p<0.001)$. Therefore, this model represents partial mediation of the effects of teacher-student relations on achievement by engagement. Note that although the need to run the model separately for each indirect effect may seem cumbersome, we chose this restriction because it simplifies the input for testing indirect effects, making webMASEM more user-friendly.

\subsection{3 | Example 1-Moderator analysis}

In the "Moderator analysis" tab, we can select the moderator variable from the dataset and select the model parameters to be tested for moderation (see screenshot 1.5 in Data S1). In our example, the moderator variable is a dummy variable for which samples from primary schools are coded 0 and samples from secondary schools are coded 1. As indicated in webMASEM, dummy variables should not be standardized in the analysis, so we unchecked this checkbox.

The list of model parameters contains direct effects (or factor loadings) only, so in this example there will be five direct effects that can be selected. The names in the list of the parameters equal the names in the output from the previous tab. We selected all five direct effects to be moderated by school type.

The output first shows the mean, standard deviation, median, minimum and maximum values of the moderator variable. In this case it shows that $41 \%$ of the studies evaluated a secondary school sample. Below, we see that the omnibus test of the five moderating effects is significant at $\alpha=0.05$, with $\chi^{2}(5)=13.548, p=0.019$, indicating that at least one of the direct effects is moderated by school type. The test on the individual parameters are shown below the omnibus test. It shows that only the effect of "pos" on "enga" is significantly moderated by school type. The regression coefficient of the moderator on the parameter equals 0.093 , meaning that on average the direct effect of "pos" on "enga" in secondary school samples is 0.093 larger than that in primary school samples. The summary from metaSEM shows all estimates from the moderation model. The parameter labeled "b31" represents the direct effect in primary school samples (for which the value on the moderator was 0) and the parameter labeled "b31_1" represents the effect of the moderator on "b31." Similarly, the parameter labeled "b43" represents the average effect for studies with value 0 on the moderator variable (primary schools here), while the parameter labeled "b43_1" represents how much "b43" is expected to change when the moderator variable changes by one unit (representing secondary schools here). When the moderating variable would have been a standardized continuous variable, a value of 0 on the moderator corresponds to the mean of the moderator, and one unit change in the moderator corresponds to one standard deviation change in the moderator.

The last part of the output shows the residual between-studies variances of the six correlation coefficients from the model with the moderator. In order to obtain the standard deviations, which are in the same metric as the correlation coefficients, one should take the square root of these variances. As explained before, these parameter estimates quantify the heterogeneity of the correlation coefficients, and not of the SEM parameters directly.

\subsection{Example analysis 2}

We illustrate fitting a factor model using webMASEM for one-stage MASEM on the dataset from Li et al. ${ }^{35}$ This dataset contains 26 correlation matrices of the Classroom Assessment Scoring System (CLASS), which is a measurement instrument consisting of 10 items about teacher-student interactions and classroom quality. Higher scores on the indicators represent more of the common factor that it measures, except for the item "NC" which is reverse coded. The hypothesized factor structure on the 10 items is shown in Figure 3.

\subsection{1 | Example 2 - Data input}

The datafile "Data_Li.dat" contains the correlation coefficients between the 10 variables, the sample sizes, and the variable "grade" that is scored 0 for studies that focused on pre-Kindergarten, Kindergarten, or preschool only and 1 for studies that (also) focused on elementary grade levels or beyond (see screenshot 2.1 in Data S1). More specific information about the studies can be found in table 3 of Li et al. ${ }^{35}$ The order of the studies in the datafile matches the ordering in Table 3.

The variable names to be used in the analysis are entered, separated by commas: "PC, NC, TS, RSP , BM, $P D, I L F, C D, Q F, L M . " ~ N e x t$, we selected the columns from the datafile containing the correlation coefficients. Since the model has 10 variables, the number of correlation coefficients equals $(10 \times 9) / 2=45$, so we need to select 45 columns. Note that the ordering of the columns in the datafile is organized as explained earlier. Next, we select the column with the sample sizes " $n$ " and the column with the moderator "grade." 
F I G URE 3 Hypothesized factor structure on 10 CLASS items. ${ }^{30}$ Abbreviations: BM, Behavior management; $\mathrm{CD}$, concept development; ILF, instructional learning formats; LM, language modeling; NC, negative climate; PC, positive climate; $\mathrm{PD}$, productivity; QF, quality of feedback; RSP, regard for student perspective; TS, teacher sensitivity

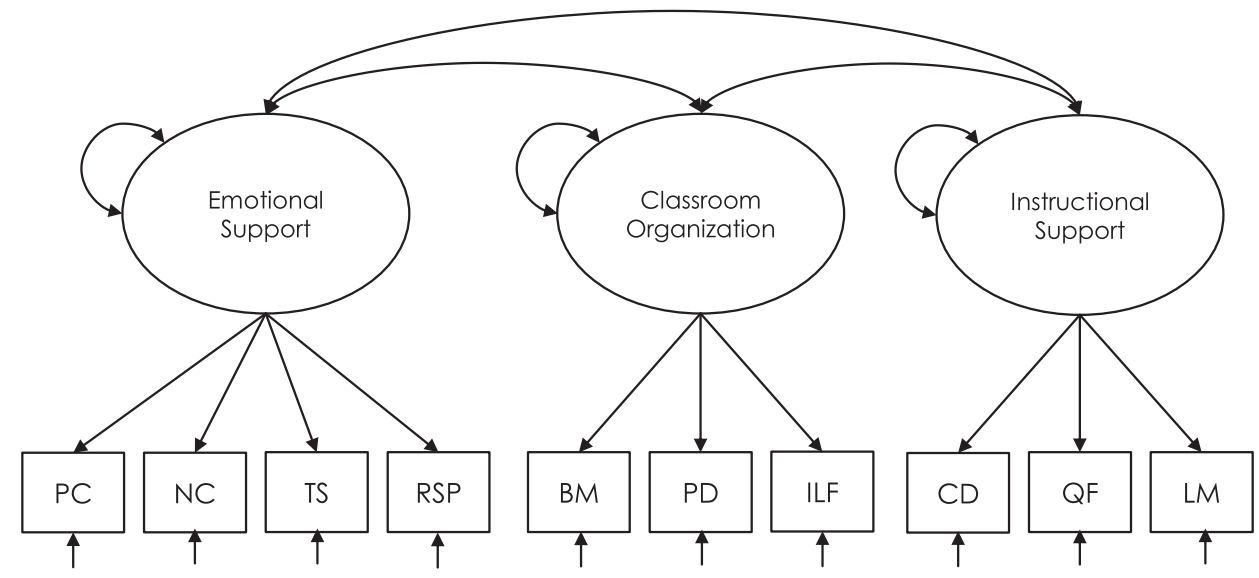

The output tab called "User dataset" shows how the dataset is read in. The "Summary" shows that all studies included the variables "PC," "NC," and "TS," while all other variables were missing in at least one study. In this dataset, all missing values are at the variable level. The total sample size for each correlation coefficient varies from 3203 for correlations involving "LM" to 5723 for correlations between the variables without missing data. As a preliminary analysis, we also estimated the average correlation matrix (see screenshot 2.2 in Data S1).

\subsection{2 | Example 2 - Overall analysis}

The three-factor model from Figure 3 was specified using the lavaan syntax provided below (see screenshot 2.3 in Data S1). Note that only the factor loadings and the factor correlations are explicitly specified. The residual variances of the indicators will be part of the MASEM and will be shown in the plot and the output. The factor variances are always fixed to one in the metaSEM package, so we do not need to fix them in the syntax.

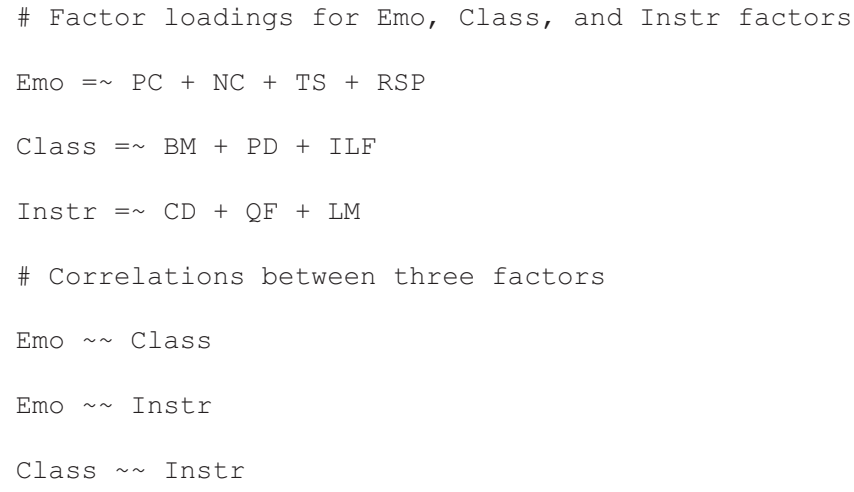

When we hit the button "Run analysis" the MASEM will be fit to the correlation matrices. Note that this is a relatively large model (i.e., there will be 45 between- studies variances estimated, on top of the 10 factor loadings and three factor covariances), therefore it takes several minutes to obtain a solution.

The output shows that there were no convergence problems and provides a plot of the model with parameter estimates. In larger models like this, the estimated factor loadings are not always readable in the plot, but we can see their values in the summary output below the model fit. The fit of the three-factor model can be read in the summary and is repeated in text as well. The chi-squared statistic is statistically significant, $\chi^{2}(32)=222.777$, $p<0.001$, indicating that the model does not fit the data exactly. Generally, we do not expect models to fit the data exactly. The Root Mean Squared Error or Approximation (RMSEA) is a measure of approximate fit, based on the idea that models should fit the data approximately. Values below 0.05 (or 0.06) are suggested to represent close approximate fit. ${ }^{36}$ The three-factor model has an RMSEAvalue of 0.032 , with $95 \%$ confidence interval running from 0.028 to 0.037 , so the confidence interval falls in the area of close approximate fit. The Standardized Root Mean Squared Residual (SRMR) represents the average correlation residual, where correlation residuals are the difference between the observed correlation matrix and the model implied correlation matrix. In one-stage MASEM the model-implied correlation matrix obtained by fitting a saturated model is used in place of the "observed" matrix. For the SRMR, values under 0.08 are taken to indicate satisfactory model fit. ${ }^{37}$ The SRMR for the three-factor model equals 0.075. Based on these fit indices we consider the model fit adequate.

The absolute values of the factor loadings range from 0.509 to 0.886 and all factor loadings are statistically significant. The correlation between the factors "Emo" and "Class" is highest with $r=0.844$, followed by the correlation between "Class" and "Instr" with $r=0.697$, and a correlation of $r=0.593$ between "Emo" and "Instr." The output further contains the estimates of the 45 betweenstudies variances of the model-implied correlations on a 
log-scale. The back-transformed variance values are shown below the summary.

The residual variances of the indicators are not a real parameter in the model. Therefore they are not part of the output in the summary of metaSEM. The residual variances are calculated by subtracting the "common variance" from the total indicator's variance, which is 1 by the definition of a correlation matrix. The common variance for each indicator in factor models without cross loadings equals the factor loading squared times the factor variance (which is 1 in MASEM). This means that. $856^{2} \times 100=73.3 \%$ of the variance in the indicator "PC" is explained by the common factor "Emo." The residual variance of the indicator "PC" equals $0.1-.856^{2}=0.267$ (rounded to two decimals in the plot). The residual variances (which equal the total variances for exogenous variables) are also displayed in the output.

\subsection{3 | Example 2- Moderator analysis}

We selected the dummy variable "grade" as the moderator variable and unchecked the tickbox for standardization of the moderator (see screenshot 2.4 in Data S1). We selected all factor loadings to be moderated by "grade." This analysis again took several minutes to run. The results show a significant overall effect of the moderator $\left(\chi^{2}(10)=46.002, p<0.001\right)$. When we look at the moderation effects on the individual parameters, it shows that this overall significant effect is mainly driven by the effect on the factor loading for "RSP" on "Emo." Compared to the factor loading in samples that focused on pre-Kindergarten, Kindergarten, or preschool only, this factor loading for "RSP" on "Emo" is estimated to be 0.327 higher in samples that involved elementary grades or beyond, meaning that RSP is more indicative (a stronger measure) of "Emo" in samples involving higher grade levels. In addition, smaller statistically significant moderating effects are found for the factor loadings of the indicators "BM" $(\hat{\beta}=-0.111)$, "PD" $(\hat{\beta}=-0.076)$, and "ILF" $(\hat{\beta}$ $=0.083$ ). The last part of the output shows the residual between-studies variances of the 45 correlation coefficients from the model with the moderator.

\section{3 $\quad$ Example analysis 3}

In the last example, we analyze the data of Nohe et $\mathrm{al}^{38}$ in webMASEM. In contrast to the previous two examples, this example involves a continuous moderator variable. Figure 4 displays the model that was hypothesized by Nohe et al. $^{38}$ The authors used MASEM to evaluate the direction and strength of the relation between work- family conflict and strain using measurement at two time points. The primary studies differed in the time lags between the measurement waves. Nohe et al were interested in whether time lag moderated the regression coefficients, expecting that the longer the period between the measurements, the weaker the effects over time.

\subsection{1 | Example 3 - Data input}

The data of the study of Nohe et $\mathrm{al}^{38}$ is already provided in webMASEM. We selected this dataset by first checking the box "Choose dataset from metaSEM" and second selecting "Nohe15A1" (see screenshot 3.1 in Data S1). This dataset consists of 32 correlation matrices with the six bivariate correlation coefficients between the strain variables at both timepoints (indicated with "S1" and "S2") and work-family conflict at both timepoints (indicated with "W1" and "W2"). The first tab of the output will remain empty in this case, because we did not read in any external datafile. By clicking the tab "Summary" one can see the order of the variables and the given names (i.e., W1, S1, W2, and S2). The summary also shows that there are no missing correlations, since the number of studies for all bivariate correlations is 32 . The total sample size for all bivariate correlation coefficients is 12,906 (see screenshot 3.2 in Data S1).

\subsection{2 | Example 3 - Overall analysis}

After clicking the tab "Overall analysis" we entered the following lavaan syntax of the hypothesized overall model (without the moderator):

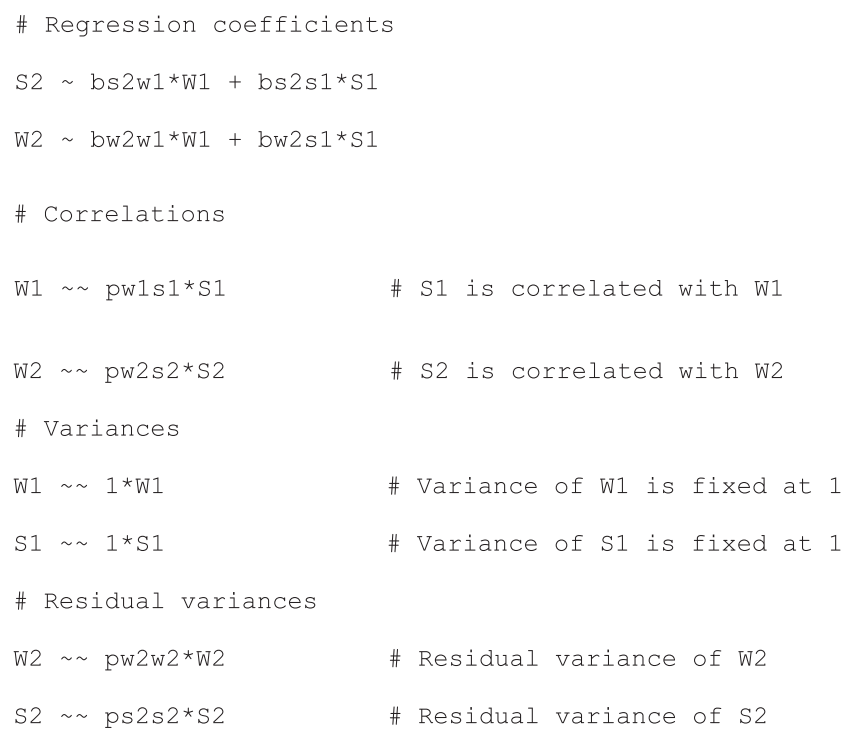


F I G U R E 4 Hypothesized path model by Nohe et al. ${ }^{38}$ [Colour figure can be viewed at wileyonlinelibrary.com]

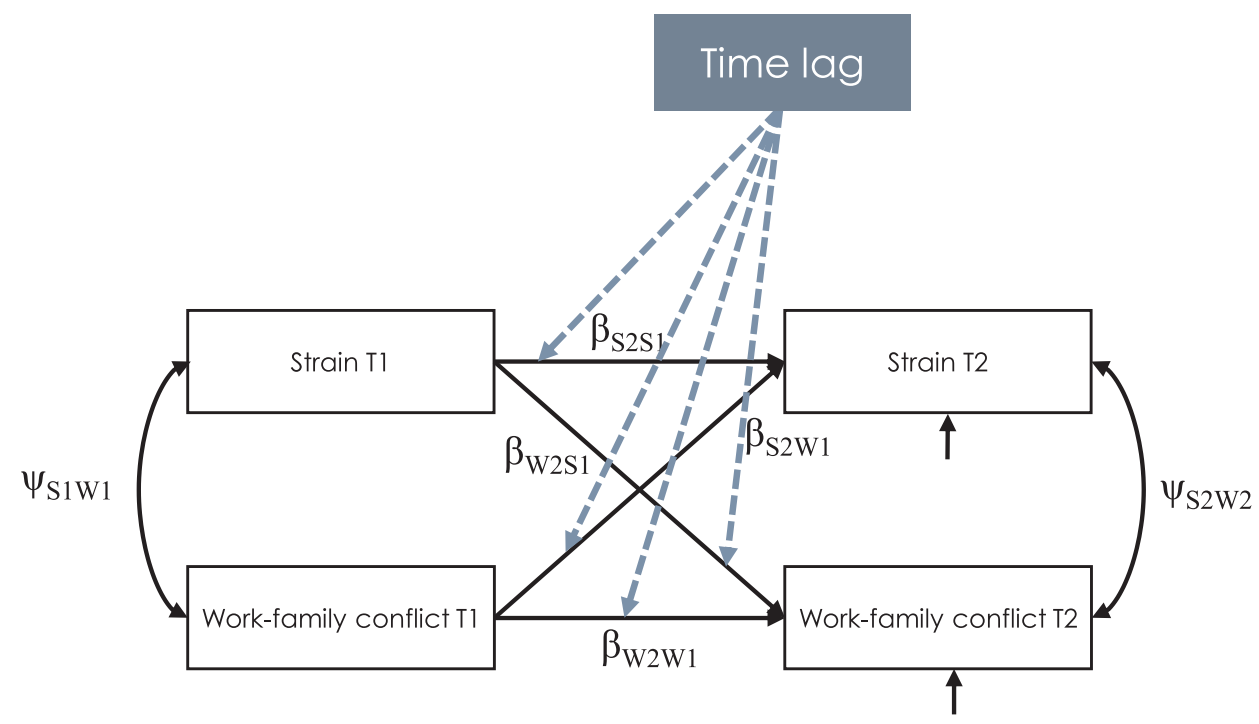

After entering the lavaan syntax, we hit the button "Run analysis" (see screenshot 3.3 in Data S1). In the output tab, we see a plot of the hypothesized model with the parameter estimates and the statement that the estimation process has converged. Since this model is saturated (i.e., the model has zero degrees of freedom), the fit statistics are not informative, but the parameter estimates, which are provided below this, are. In the summary provided by metaSEM, we see, for example, statistically significant positive direct effects of the same variable over time $\left(\hat{\beta}_{w 2 w 1}\right.$ $=0.572, p<0.001$ and $\hat{\beta}_{s 2 s 1}=0.589, p<0.001$ ). The two cross-lagged effects are both positive, statistically significant, and of similar size $\left(\hat{\beta}_{\text {s2w1 }}=0.080, p=0.001\right.$ and $\left.\hat{\beta}_{w 2 s 1}=0.086, p<0.001\right)$.

The output further shows that the residual variance in W2 equals 0.628 , meaning that the proportion of explained variance equals $0.1-0.628=0.372$. Similarly, the proportion of explained variance in S2 equals 0.1-0.614 $=0.386$.

The last part of the output shows the between-studies variances of the six model implied correlation coefficients. Note that these between-studies variances do not quantify the heterogeneity of the direct effects in the path model, but the heterogeneity of a function of the SEM parameters. One can see how the SEM parameters together form the model implied correlation coefficients by clicking the button under the lavaan syntax that says "View model implied matrix."

\subsection{3 | Example 3- Moderator analysis}

Since we are interested in evaluating whether there is a moderator effect of time lag on the regression coefficients between all variables, we select "lag" in the field "Select the moderator" and select all four regression coefficients to be moderated (i.e., bw2w1, bs2w1, bw2s1, and bs2s1). We check the box "Standardize the moderator" because time lag is a continuous variable, and the variable needed to be rescaled in order to obtain a converged solution (see screenshot 3.4 in Data S1). After clicking the button "Run analysis," we get the output for the moderation analysis. At the top of the output, we see that there are no convergence problems. Underneath, we see the summary statistics of the moderator. The mean time lag between the measurement waves is 14.12 months, the median value is 12 months, the standard deviation is 16.36 months, and time lag ranges from 0.30 months to 72 months. The omnibus test of the four moderating effects indicates that at least one of the direct effects is moderated by time lag $\left(\chi^{2}(4)=23.522, p<0.001\right)$.

The output provided under "Individual moderating effects" indicates which of the effect(s) is/are significantly moderated by time lag. We can see that time lag only significantly moderates the effect of W1 on W2. The "Summary of Moderation by Lag" provides all estimates from the moderation model. The parameter with the name "bw2w1" here represents the intercept of the direct effect of W1 on W2 (i.e., $\hat{\beta}_{w 2 w 1}=-0.573, p<0.001$ ). In other words, "bw2w1" here represents the estimated direct effect of W1 on W2 in studies for which the moderator was 0 . As the moderator was standardized, this pertains to studies with a time lag of 14.12 months (the mean time lag). The parameter with the name "bw2w1_1" represents how much the effect W1 on W2 is expected to change when the moderator variable increases by one standard deviation. The corresponding regression coefficient is estimated as $\hat{\beta}_{w 2 w 1 \_1}=-0.062, p<0.001$, which means that for every standard deviation (i.e., 16.36 months) increase in time lag it is expected that the effect of W1 on W2 decreases by 0.062 points. At the 
bottom of the output of the tab "Moderator analysis," you can find the residual between-studies variances of all correlation coefficients of the model with the moderator.

\section{3 | DISCUSSION}

In this article, we presented a tutorial and an online application to facilitate one-stage MASEM. When designing webMASEM we aimed at finding a good balance between providing enough functionality, while keeping the application user friendly and intuitive in use. There are situations in which webMASEM is not the appropriate software. Some of these situations are discussed below.

\section{1 | Features that are not implemented in webMASEM}

As illustrated in the examples, the moderation of regression coefficients (direct effects in a path model or factor loadings in a factor model) is implemented in webMASEM. One-stage MASEM also allows the moderation of covariance parameters, such as the covariance between two outcome variables in a path model or the correlation between two common factors in a factor model. In our experience, researchers' hypotheses almost always concern moderation effects on regression coefficients, and not on covariances. However, researchers who are interested in evaluating moderating effects on covariance parameters can do so using the osmasem() functions from the metaSEM package directly.

webMASEM also does not allow for the evaluation of multiple moderator variables at the same time. That means that researchers cannot estimate the effect of one moderator, controlled for the effect of another moderator. The osmasem() functions from the metaSEM package do allow such an analysis. It is, however, recommended to keep the analyses as simple as possible, by limiting the number of moderators as well as the number of parameters to be moderated. ${ }^{11}$ Another reason that researchers will probably evaluate moderators one at a time, is that studies with missing values on one or both of the moderators should be deleted from the dataset before conducting the moderation analysis. With two moderator variables with potential missing values, one will also have to delete studies that have a missing value on only one of the moderator variables.

webMASEM returns an error if users attempt to analyze a moderator variable with missing values. This means that users will have to delete the studies with missing values on the moderating variable from the dataset, before conducting the overall and moderator analyses (again). We considered automatic deletion of studies with missing values on the moderator, but chose not to implement this, so that users are aware which studies are deleted.

As explained in the introduction, one-stage MASEM, and consequently webMASEM, does not quantify the heterogeneity of the SEM parameters. Instead, one obtains estimates of the between-studies variances of the correlation coefficients. There exist other approaches to obtain heterogeneity estimates of SEM parameters itself. These methods are Bayesian MASEM, ${ }^{9}$ Full Information MASEM,${ }^{39-41}$ or the direct synthesis of regression slopes. ${ }^{42}$

Any program with a graphical user interface will be kind of a black box. Users may be able to use webMASEM without knowing what is exactly happening behind the scenes. The downloadable reports that are available are meant to provide the user a savable overview of the results, but also to remedy this problem. The reports contain all necessary information to replicate the analysis using metaSEM directly if needed. We provide the syntax for the analysis of the three examples using metaSEM directly at the OSF page related to this article: https://osf.io/wh6d3/.

\section{2 | Errors and warnings from webMASEM}

With this tutorial paper, we hope that we provided users with the necessary information to apply one-stage MASEM to their data. In practice, we expect that users may still encounter some warnings or error messages when using webMASEM, simply because there are many ways in which setting up the analysis can go wrong. We are not able to foresee all problems, but in our experience, many issues can be solved by a careful evaluation of each step taken in the analysis. When encountering an error, the first two things to check would be the organization of the dataset and whether the dataset was read in correctly. After verifying that the data were read in correctly, one could try estimating the unrestricted average correlation matrix. If this works properly, but fitting the SEM model leads to an error, there may be problems with the lavaan syntax used to formulate the SEM model. Common mistakes are forgetting to specify parameters, forgetting to fix the variances of exogenous variables to one, making typos in the variables' names, or just specifying the model incorrectly. If the data were read in correctly and the lavaan syntax was correct, one may still encounter convergence errors. Convergence errors will be clearly shown in the output by webMASEM by the message "No convergence..., maybe rerunning the model helps". In such a case, users are advised to click the rerun button to increase the number of attempts of fitting the model. Rerunning the analysis this way will also possibly fix between-studies variances that hindered model convergence to zero. 
If these options do not solve the problem, our advice is to run the analysis in $\mathrm{R}$, using the metaSEM package directly. This will provide more insight in the possible causes of the problems and will provide the user with more flexibility in running the analysis, since all function of the metaSEM-package can be used. We provide the scripts to run the example analyses in this manuscript at the associated OSF page.

\section{3 | Conclusion}

We presented a tutorial and Shiny app for one-stage MASEM. It is our hope that the current manuscript will be useful for researchers who need to apply one-stage MASEM.

\section{CONFLICT OF INTEREST}

The authors declare no conflict of interest.

\section{AUTHOR CONTRIBUTION}

Conceptualization: Suzanne Jak. Methodology: Suzanne Jak and Mike W.-L. Cheung. Creating webMASEM: Suzanne Jak. Testing webMASEM: Hongli Li, Laura Kolbe, Hannelies de Jonge, and Mike W.-L. Cheung. Providing data or analyses for examples: Hongli $\mathrm{Li}$ and Hannelies de Jonge. Writing-original draft preparation: Suzanne Jak. Writing—review and editing: Hongli Li, Laura Kolbe, Hannelies de Jonge, and Mike W.-L. Cheung. All authors have read and agreed to the published version of the manuscript.

\section{DATA AVAILABILITY STATEMENT}

All datasets, $\mathrm{R}$ scripts, and the source code for webMASEM are available from the OSF page of this article: https://osf.io/wh6d3/.

\section{ORCID}

Suzanne Jak (1) https://orcid.org/0000-0002-2223-5594 Mike W.-L. Cheung (i) https://orcid.org/0000-0003-01130758

\section{ENDNOTES}

* Column-major order is the default way to fill matrices in R.

$\dagger$ It is common practice in SEM to use a significance level of $\alpha=0.05$ to evaluate the statistical significance of individual parameters. However, Cribbie ${ }^{32}$ explains how Type 1 error control may be applied in SEM.

¥ In practice, for dichotomous moderators, one could fit the model to the two subgroups of studies to evaluate the fit in the two groups separately using the metaSEM package directly, provided that the number of studies per subgroup is still sufficient to fit the MASEM. $^{18}$
$\S$ Funder and $\mathrm{Ozer}^{34}$ identified univariate regression coefficients of respectively $0.10,0.20$ and 0.30 as representing small, moderate, and large effects. Indirect effects are the product of two regression coefficients. We evaluated the size of indirect effects considering indirect effects consisting of two small, two moderate, or two large effects. This procedure leads to values of $0.10^{2}=0.01$ (small), $0.20^{2}=0.04$ (moderate), and $0.30^{2}=0.09$ (large)

\section{REFERENCES}

1. Cheung MW-L. metaSEM: an R package for meta-analysis using structural equation modeling. Front Psychol. 2015;5. https://doi.org/10.3389/fpsyg.2014.01521

2. Epskamp S. semPlot: unified visualizations of structural equation models. Struct Equ Model Multidiscip J. 2015;22(3):474483. https://doi.org/10.1080/10705511.2014.937847

3. Becker BJ. Using results from replicated studies to estimate linear models. J Educ Behav Stat. 1992;17(4):341-362. https://doi. org/10.3102/10769986017004341

4. Becker BJ. Corrections to "using results from replicated studies to estimate linear models.”. J Educ Behav Stat. 1995;20(1):100102. https://doi.org/10.2307/1165390

5. Becker BJ, Aloe AM. Model-based meta-analysis and related approaches. In: Cooper HM, Hedges LV, Valentine JC. eds, The handbook of research synthesis and meta-analysis. 2019; 339-363. Russell Sage Foundation.

6. Cheung MW-L, Chan W. Meta-analytic structural equation modeling: a two-stage approach. Psychol Methods. 2005;10(1): 40-64. https://doi.org/10.1037/1082-989X.10.1.40

7. Cheung MW-L. Fixed- and random-effects meta-analytic structural equation modeling: examples and analyses in R. Behav Res Methods. 2014;46(1):29-40. https://doi.org/10.3758/s13428-013-0361-y

8. Cheung MW-L. Meta-Analysis: A Structural Equation Modeling Approach. Chichester, UK: John Wiley \& Sons; 2015.

9. Ke Z, Zhang Q, Tong X. Bayesian meta-analytic SEM: A onestage approach to modeling between-studies heterogeneity in structural parameters. Struct Equ Model Multidiscip J. 2019;26 (3):348-370. https://doi.org/10.1080/10705511.2018.1530059

10. Viswesvaran C, Ones DS. Theory testing: combining psychometric meta-analysis and structural equations modeling. Pers Psychol. 1995;48(4):865-885. https://doi.org/10.1111/j.17446570.1995.tb01784.x

11. Jak S, Cheung MW-L. Meta-analytic structural equation modeling with moderating effects on SEM parameters. Psychol Methods. 2020;25(4):430-455. https://doi.org/10. 1037/met0000245

12. van Dijk R, van der Valk IE, Deković M, Branje S. A meta-analysis on interparental conflict, parenting, and child adjustment in divorced families: examining mediation using meta-analytic structural equation models. Clin Psychol Rev. 2020;79:101861. https://doi.org/10.1016/j.cpr.2020.101861

13. Agelink van Rentergem JA, de Vent NR, Schmand BA, Murre JMJ, Staaks JPC, Huizenga HM. The factor structure of cognitive functioning in cognitively healthy participants: a meta-analysis and meta-analysis of individual participant data. Neuropsychol Rev. 2020;30(1):51-96. https://doi.org/10.1007/s11065-019-09423-6

14. Bresin K. Impulsivity and aggression: a meta-analysis using the UPPS model of impulsivity. Aggression Violent Behav. 2019;48: 124-140. https://doi.org/10.1016/j.avb.2019.08.003 
15. Norton S, Cosco T, Doyle F, Done J, Sacker A. The Hospital Anxiety and Depression Scale: a meta confirmatory factor analysis. J Psychosom Res. 2013;74(1):74-81. https://doi.org/10.1016/ j.jpsychores.2012.10.010

16. Topa G, Moriano JA. Theory of planned behavior and smoking: meta-analysis and SEM model. Subst Abuse Rehabil. 2010;1:2333. https://doi.org/10.2147/SAR.S15168

17. Cheung MW-L, Cheung SF. Random-effects models for metaanalytic structural equation modeling: review, issues, and illustrations. Res Synth Methods. 2016;7(2):140-155. https://doi.org/ 10.1002/jrsm.1166

18. Jak S, Cheung MW-L. Testing moderator hypotheses in metaanalytic structural equation modeling using subgroup analysis. Behav Res Methods. 2018;50(4):1359-1373. https://doi.org/10. 3758/s13428-018-1046-3

19. MacCallum RC, Zhang S, Preacher KJ, Rucker DD. On the practice of dichotomization of quantitative variables. Psychol Methods. 2002;7(1):19-40. https://doi.org/10.1037/1082-989X.7.1.19

20. Furlow CF, Beretvas SN. Meta-analytic methods of pooling correlation matrices for structural equation modeling under different patterns of missing data. Psychol Methods. 2005;10(2):227-254. https://doi.org/10.1037/1082-989X.10.2.227

21. Reich J, Gehlbach H, Albers CJ. "Like upgrading from a typewriter to a computer": registered reports in education research. AERA Open. 2020;6(2):1-6. https://doi.org/10.1177/ 2332858420917640

22. Rosseel Y. Lavaan: an R package for structural equation modeling. J Stat Softw. 2012;48(1):1-36. https://doi.org/10.18637/jss.v048.i02

23. Jak S. Meta-Analytic Structural Equation Modelling. New York, NY: Springer International Publishing; 2015. doi:10.1007/9783-319-27174-3

24. Bauer DJ. A more general model for testing measurement invariance and differential item functioning. Psychol Methods. 2017;22(3):507-526. https://doi.org/10.1037/met0000077

25. Boker S, Neale $M$, Maes $H$, et al. OpenMx: an open source extended structural equation modeling framework. Psychometrika. 2011;76(2):306-317. https://doi.org/10.1007/s11336-010-9200-6

26. Chang W, Cheng J, Allaire J, Xie Y, McPherson J. Shiny: web application framework for R. R package version, 1(5). http:// CRAN.R-project.org/package $=$ shiny.

27. R Core Team. R: A Language and Environment for Statistical Computing. Vienna, Austria: R Foundation for Statistical Computing; 2020. Retrieved from https://www.R-project.org/

28. Cheung MW-L. SymSEM: Symbolic Computation for Structural Equation Models. 2020. https://CRAN.R-project.org/package= symSEM. Accessed November 16, 2020

29. Roorda DL, Koomen HMY, Spilt JL, Oort FJ. The influence of affective teacher-student relationships on students' school engagement and achievement: a meta-analytic approach. Rev Educ Res. 2011;81(4):493-529. https://doi.org/10.3102/003 4654311421793

30. Neale MC, Miller MB. The use of likelihood-based confidence intervals in genetic models. Behav Genet. 1997;27(2):113-120. https://doi.org/10.1023/A:1025681223921

31. Cheung MW-L. Comparison of methods for constructing confidence intervals of standardized indirect effects. Behav Res Methods. 2009;41(2):425-438. https://doi.org/10.3758/ BRM.41.2.425
32. Cribbie RA. Multiplicity control in structural equation modeling. Struct Equ Model Multidiscip J. 2007;14(1):98-112. https:// doi.org/10.1080/10705510709336738

33. Roorda DL, Jak S, Zee M, Oort FJ, Koomen HMY. Affective teacher-student relationships and students' engagement and achievement: a meta-analytic update and test of the mediating role of engagement. Sch Psychol Rev. 2017;46(3):239-261. https://doi.org/10.17105/SPR-2017-0035.V46-3

34. Funder DC, Ozer DJ. Evaluating effect size in psychological research: sense and nonsense. Adv Methods Pract Psychol Sci. 2019;2(2):156-168. https://doi.org/10.1177/2515245919847202

35. Li H, Liu J, Hunter CV. A meta-analysis of the factor structure of the classroom assessment scoring system (CLASS). J Exp Educ. 2020;88(2):265-287. https://doi.org/10.1080/00220973.2018.1551184

36. MacCallum RC, Browne MW, Sugawara HM. Power analysis and determination of sample size for covariance structure modeling. Psychol Methods. 1996;1(2):130-149. https://doi.org/ 10.1037/1082-989X.1.2.130

37. Hu L, Bentler PM. Cutoff criteria for fit indexes in covariance structure analysis: conventional criteria versus new alternatives. Struct Equ Model Multidiscip J. 1999;6(1):1-55. https:// doi.org/10.1080/10705519909540118

38. Nohe C, Meier LL, Sonntag K, Michel A. The chicken or the egg? A meta-analysis of panel studies of the relationship between work-family conflict and strain. J Appl Psychol. 2015; 100(2):522-536. https://doi.org/10.1037/a0038012

39. Yu J(J), Downes PE, Carter KM, O'Boyle EH. The problem of effect size heterogeneity in meta-analytic structural equation modeling. J Appl Psychol. 2016;101(10):1457-1473. https://doi. org/10.1037/apl0000141

40. Cheung MW-L. Issues in solving the problem of effect size heterogeneity in meta-analytic structural equation modeling: a commentary and simulation study on Yu, Downes, Carter, and O'Boyle (2016). J Appl Psychol. 2018;103(7):787-803. https://doi. org/10.1037/apl0000284

41. Yu J(J), Downes PE, Carter KM, O'Boyle E. The heterogeneity problem in meta-analytic structural equation modeling (MASEM) revisited: a reply to Cheung. J Appl Psychol. 2018; 103(7):804-811. https://doi.org/10.1037/apl0000328

42. Becker BJ, Wu M-J. The synthesis of regression slopes in metaanalysis. Stat Sci. 2007;22(3):414-429. https://doi.org/10.1214/ 07-STS243

\section{SUPPORTING INFORMATION}

Additional supporting information may be found online in the Supporting Information section at the end of this article.

How to cite this article: Jak S, Li H, Kolbe L, de Jonge H, Cheung MW-L. Meta-analytic structural equation modeling made easy: A tutorial and web application for one-stage MASEM. Res Syn Meth. 2021;12(5):590-606. https://doi.org/10.1002/ jrsm.1498 